\title{
New Small Wheel Trigger Simulation and Performance
}

\author{
Chilufya Mwewa*, on behalf of the ATLAS Muon collaboration \\ University of Cape Town, Cape Town, South Africa \\ E-mail: chilufya.mwewa@cern.ch
}

\begin{abstract}
The instantaneous luminosity of the Large Hadron Collider (LHC) at CERN will be increased up to a factor of seven with respect to the original design value to explore higher energy scales. In order to benefit from the expected High Luminosity (HL) performance, the first station of the ATLAS muon end-cap system will be replaced by a New Small Wheel (NSW) detector. The NSW consists of two types of detector technologies; MicroMesh gaseous detectors (MM) and small-strip Thin Gap Chambers (sTGC) both with good tracking and triggering capabilities. The NSW will provide precise track-segment information to the muon Level-1 trigger system and hence reduce fake muon triggers. This article summarizes the simulation of the NSW trigger decision system, the track reconstruction algorithm implemented into the trigger processor and results of performance studies on the trigger system showing that the NSW will perform well in high luminosity conditions.
\end{abstract}

The 39th International Conference on High Energy Physics (ICHEP2018)

4-11 July, 2018

Seoul, Korea

${ }^{*}$ Speaker. 


\section{NSW Trigger System and Algorithm}

The muon end-cap L1 trigger is provided by a hit coincidence, in terms of $(\eta, \phi)$, between the present SW and the Big Wheel (BW) TGC but does not effectively reject fake muons which do not originate from the interaction point [1]. The NSW will provide more precise measurements and will measure a third parameter, $\Delta \theta$ to reject fake muon triggers. Each MM layer has four layers of horizontal readout strips (X) and four layers of stereo strips (UV). Once a strip registers a hit, a multiplane hit coincidence is used to find a particular track segment by requiring a minimum number of $\mathrm{X}$ and UV planes to be hit, after which $(\eta, \phi)$ and $\Delta \theta$ parameters are calculated [2]. On the other hand, an sTGC layer consists of two readout electrodes, each having either pads or strips and each pad has four towers. A 3 out of 4 coincidence of pad towers is used to define regions of interest. Information from strips that correspond to these regions is then read out and transmitted to the trigger processor. $(\eta, \phi)$ and $\Delta \theta$ parameters are finally calculated and merged with that of the MM.

\section{NSW Trigger Performance}

Performance of both MMs and sTGCs in simulation is determined by their track finding efficiencies. Performance plots are shown in Figure 1. For MMs, good efficiency is observed for 2X2UV and 3X3UV coincidences where as for sTGCs, a very good efficiency of $99 \%$ is generally observed.
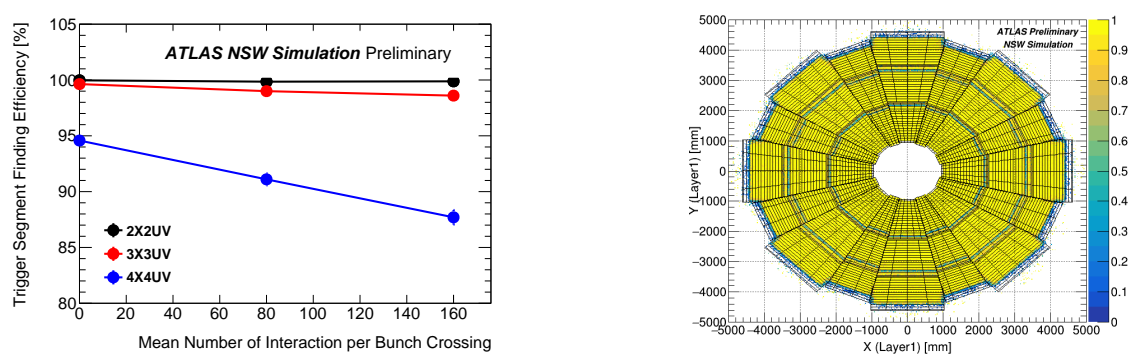

Figure 1: Left: MM track finding efficiencies as a function of $\mu$ (pileup). Right: Trigger efficiency of sTGC pivot quadruplet pads projected in the transverse plane.

\section{Conclusion}

Software for the complete simulated study of the NSW has been developed and offers deep insight on the NSW trigger logic. Performance studies have shown that the NSW trigger system is capable of working with very good performance as required for the high luminosity LHC.

\section{References}

[1] ATLAS Collaboration, 2013 New Small Wheel Technical Design Report (CERN-LHCC-2013-006), https://cds.cern.ch/record/1602235/

[2] T.Saito, on behalf of the ATLAS Collaboration, 2017 PoS (TWEPP-17) 115 